\title{
Chicago Exploration Days
}

\author{
E. Zeitler
}

Fritz-Haber Institute, Max-Planck Society, Berlin, Germany

Department of Inorganic Chemistry, zr@fhi-berlin.mpg.de

When I received the invitation for this contribution to the Albert Crewe Symposium I accepted it with delight and thanks; I was always eager to explore the conditions and ingredients indispensable for the origin of such a supernova here in electron microscopy. I shall present my conjectures in a most personal way. I had met Albert for the first time in 1966 at a workshop on High-Voltage Electron Microscopy that the Argonne National Lab had sponsored together with the Associated Midwestern Universities. At this time the 39 year old Albert had been director of this Lab with 5000 people for five years; in the proceedings one can find a description of a field-emission gun-lens, which Albert had ordered at Applied Mathematic. Jim Butler then came up with a successful design henceforth called the Butler gun. The participants were also shown an impressive STEM under construction depicted in Fig. 2 of Isaacson's contribution. Here we meet also another essential component, namely enthusiastic students, engaged and curious. Albert, the Professor with his ideas and microscopes and with his outstanding students, Joe Wall, Mike Isaacson and Dale Johnson moved to the Department of Physics of the UofC where they could reassess their plans of future developments without the distraction of administrative duties.

In 1938, von Ardenne built a scanning transmission electron microscope using a conventional column. The 20 minute exposure time required for a single picture was acceptable because Raman recording could take hours and was also accepted, said von A. It was, however, the contamination which made meaningful microscopy impossible. In the light of this, Albert looks for a solution by a novel approach, he uses his fantasy and also his knowledge from accelerator construction whose techniques are at the cutting edge. When the exposure-time of a single pixel can be only one millisecond, I need a very bright source and when contamination is the hindrance I need a superclean Hi-Vacuum: so a cold field emitter is the choice; they were around at the time, but for microscopic use they were too unstable and too fickle - they had to be tamed, by the students. This example is typical for the modus operandi of the group.

The Field-Emission-Microscope is so small that it can be mounted directly onto the sturdy and heavy ion-pump; this simple gun microscope invites a systematic development of accessories like a magnetic spectrometer. Indeed, this was the instrument on which Mike and Dale earned their PhD's and with their important results on Electron-Energy-Loss-Spectroscopy (EELS) they found entrance into Academia. 


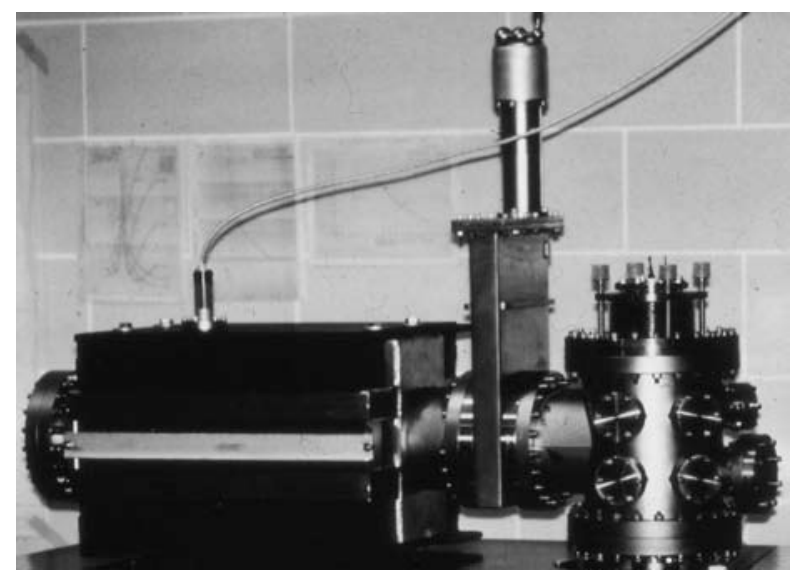

M.S. Isaacson and D. Johnson, Simple Scanning Electron Microscope, Rev.Sci.Inst. 40, 241, (1969)

Mike: Narinder Kapany Professor of Electrical Engineering, UCSC

Dale: Professor of Physics. U of South Florida Joe Wall: PhD in Biophysics U of C 1971, 5 Angstrom STEM, Senior Biophysicist, Head of the user STEM-facility he established at Brookhaven Labs

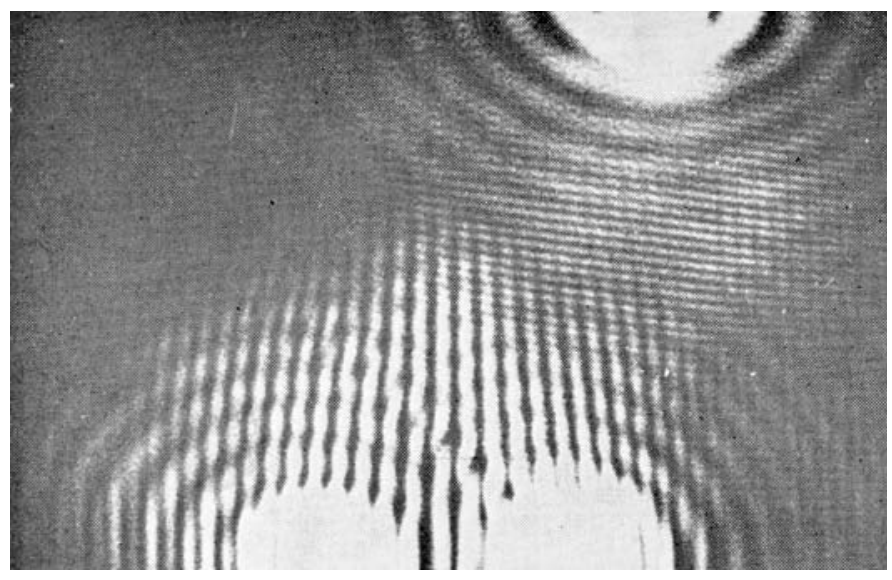

This picture, taken from Jesper Munch's thesis and analogous to a Young double slit interference pattern, was produced by electrons passing through minute holes in a gold film.

J. Munch Experimental Electron Holography, Optik 40, 79, (1975)

Jesper: Professor of Physics, U Adelaide, current Chair of the Australian Consortium for Gravitational Astronomy

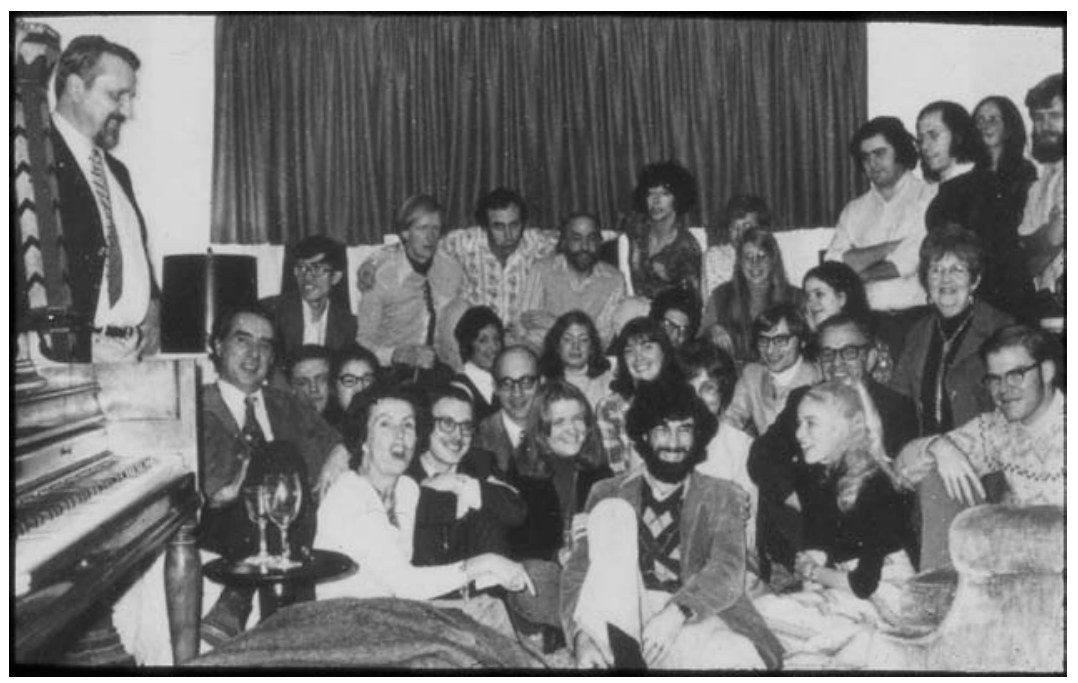

There is one more requirement for a fruitful collaboration, a human one and perhaps the most important one: the chemistry must be right! This is demonstrated by the snapshot of the group at an occasional gathering:

The spouses and the girlfriends know each other and are welcomed guests, Albert and his wife Doreen are present, as is one most important person, Albert's secretary, Judith Reiffel, who was the good fairy at the heart of the group. 